\title{
Demographic, Clinical, and Angiographic Characteristics of Atrial Fibrillation Patients Suffering from De Novo Acute Myocardial Infarction
}

\author{
Ali Coner ${ }^{1}$, Cenk Ekmekci ${ }^{1}$, Gokhan Aydin ${ }^{1}$, Yasemin Dogan ${ }^{1}$, Ozlem Arican Ozluk ${ }^{1}$, Salih \\ Kilic $^{1}$, Yunus Celik ${ }^{1}$, Ismail Ungan ${ }^{1}$, Mustafa Begenc Tascanov ${ }^{1}$, Ramazan Duz ${ }^{1}$, Veli \\ Polat $^{1}$, Hakan Ozkan ${ }^{1}$, Mehmet Ozyasar ${ }^{1}$, Kamil Tuluce ${ }^{1}$, Abdullah Icli $^{1}$, Devrim Kurt ${ }^{1}$, \\ Nurullah Cetin ${ }^{1}$, Murat Gul ${ }^{1}$, Sinan Inci ${ }^{1}$, Fatma Yilmaz Coskun ${ }^{1}$, Ayse Akdeniz ${ }^{1}$, Mehdi \\ Zoghi $^{2}$, Oktay Ergene ${ }^{1}$, and Ugur TURK ${ }^{3}$ \\ ${ }^{1}$ Affiliation not available \\ ${ }^{2}$ Ege University \\ ${ }^{3}$ Central Hospital
}

November 22, 2020

\begin{abstract}
Abstract Aim: The prevalence of atrial fibrillation (AF) in patients with myocardial infarction (MI) ranges widely and has been reported to be as high as $21 \%$. However, the demographic, clinical, and angiographic characteristics of AF patients with de novo MI is unclear. The aim of this study was to investigate the prevalence of patients presenting with de novo MI with AF. Methods: The study was performed as a sub-study of the MINOCA-TR (Myocardial Infarction with Non-obstructive Coronary Arteries in Turkish Population) Registry, a multicenter, cross-sectional, observational, all-comer registry. MI patients without a known history of stable coronary artery disease and/or prior coronary revascularization were enrolled in the study. Patients were divided into AF and Non-AF groups according to their presenting cardiac rhythm. Results: A total of 1793 patients were screened and 1626 were included in the study. Mean age was 61.5 (12.5) years. $70.7 \%$ of patients were men. Prevalence of AF was $3.1 \%$ (51 patients). AF patients were older [73.4 (9.4) vs. 61.0 (12.4) years, $\mathrm{p}<0.001]$ than non-AF patients. The proportion of women to men in the AF group was also higher than in the non-AF group ( $43.1 \%$ vs. $28.7 \%$, p=0.027). Dramatically, the minority of patients were previously diagnosed with AF (14 patients, 27.4\%) and only 1 out of every 5 AF patients (10 patients, $19.6 \%$ ) was using oral anticoagulants (OAC). Conclusions: AF prevalence in patients presenting with de novo MI was lower than reported in previous studies. The majority of AF patients did not have any knowledge of their arrhythmia and were not undergoing OAC therapy at admission, emphasizing the vital role of successful diagnostic strategies, patient education, and implementations for guideline adaptation.
\end{abstract}

\section{Introduction}

Atrial fibrillation (AF) is a common arrhythmia in the general population and ranks third in incidence in patients with myocardial infarction (MI) after sinus bradycardia and sinus tachycardia. ${ }^{1}$ Previous studies have reported a wide variety in the prevalence rates of AF in the MI population. European Society of Cardiology (ESC) 2017 Guidelines for the management of acute myocardial infarction in patients presenting with ST-segment elevation (STEMI) reported prevalence rates as high as $21 \%$ for MI patients. ${ }^{2} \mathrm{AF}$ can result in undesired in-hospital outcomes such as recurrent coronary ischemia, congestive heart failure, or stroke, as well as the need for long-term oral anticoagulation use, which increases rates of bleeding complications. Preexisting, known coronary artery disease (CAD) is a well-recognized comorbidity in patients with AF. CAD 
and other comorbidities carry the risk for incident AF. Interestingly, there is scarce demographic and clinical information in the literature on AF in the MI population without a history of CAD and coronary revascularization. Evidence for acute management and long-term treatment strategies such as oral anticoagulation are not clear and should be identified. ${ }^{3}$

This study aimed to determine prevalence rates of AF in de novo MI patients without revascularization history and to compare the demographic, clinical, and angiographic characteristics of patients with and without AF in the de novo MI population in Turkey.

\section{Methods}

Study design:

The registry of Myocardial Infarction with Non-obstructive Coronary Arteries in the Turkish population (MINOCA-TR) is a nationwide, multicenter, prospective, and observational cohort study of patients with a de novo myocardial infarction (MI) presentation. A total of 32 invasive cardiology centers (eighteen university, ten state, and four private hospitals) around Turkey participated to the study. Patient recruitment was performed between March to June 2018. Patients without a known history of prior coronary revascularization who underwent a diagnostic coronary angiography procedure with a possible diagnosis of MI were screened and included in the study population. The aim of this screening was to obtain real-life data and prevent potential bias in the clinical context. A sub-study of the MINOCA-TR registry, this paper focuses on the prevalence of AF in the MINOCA-TR study population. ${ }^{4}$ The study population was divided into two groups, the AF and non-AF groups, based on their presenting rhythm at admission. Demographic, clinical, and angiographical data of the MINOCA-TR study population was recorded and compared. The study protocol of the MINOCA-TR was approved by the Clinical Research Ethical Committee of Dokuz Eylul University and was registered withwww.clinicaltrials.gov(NCT03364387).

\section{Study population:}

Consecutive patients 18 years of age and older who were diagnosed with MI using the Third Universal Definition of Myocardial Infarction and who underwent a diagnostic coronary angiography were included in the study population. Each participating physician arranged the management of treatment on their own, and the study patients were not given any medical procedure other than guideline directed therapy. Potential study patients were informed about the study and asked to sign informed consent forms after coronary angiography (or percutaneous coronary interventions if needed). Patients with stable coronary artery disease, unstable angina pectoris, coronary revascularization history (PCI or CABG), or type 4 or type $5 \mathrm{MI}$ and patients under the years of 18 and who did not sign the informed consent were excluded from the study.

\section{Coronary angiographies:}

Coronary angiographies were performed in the individual centers involved in the study. A digital copy of each recording was sent to the MINOCA-TR adjudication committee, which was made up of three invasive cardiologists. Coronary angiographies were evaluated for the possible presence of an overlooked type $1 \mathrm{MI}$.

\section{Statistical methods:}

Statistical analysis was descriptive, exploratory, and generally limited to frequency or summary statistics (e.g., means \pm standard deviation or medians \pm quartiles). Demographic information was summarized according to the type of data by descriptive statistics (n, mean, standard deviation, median, the difference between percentiles) or frequency distribution (n and \%). The Student's t-test was used for continuous variables, the analysis of variance for categorical variables, and the Pearson's chi-square test for binominal variables. $\mathrm{P}$ values of $<0.05$ were accepted as significant.

\section{Results}

Baseline characteristics of study population: 
A total of 1793 patients were screened for eligibility. Of these, 1626 patients admitted with de novo acute MI were enrolled in this study. The mean (SD) age of the population was 61.5 (12.5) years and men made up $70.7 \%$ of the study population. Fifty-one $(3.1 \%)$ patients were diagnosed with AF. Dramatically, the minority of patients were previously diagnosed with AF (14 patients, 27.4\%) and only 1 out of every $5 \mathrm{AF}$ patients (10 patients, 19.6\%) was using oral anticoagulants (OAC). Demographic and clinical characteristics of the study population are presented in Table 1. Patients in the AF group were older than the non-AF group [mean 73.4 (9.4) vs. 61.0 (12.4) years, $\mathrm{p}<0.001$ ]. The percentage of women in the AF group was also higher than that in the non-AF group ( $43.1 \%$ vs. $28.7 \%, \mathrm{p}=0.027)$. Non-ST segment elevation MI (NSTEMI) presentation $(68.6 \%$ vs. $53.2 \%, \mathrm{p}=0.023)$ and prevalence of hypertension $(64.7 \%$ vs. $48.5 \%, \mathrm{p}=0.023)$ were more common in patients in the AF group. Conversely, fewer patients in the AF group smoked than in the non-AF group (39.2\% vs. $58.6 \%, \mathrm{p}=0.006)$. Median LV ejection fraction at admission was lower in $\mathrm{AF}$ patients than the non-AF patients [45.0\% (30-50\%) vs. 50.0\% (35-55\%), p=0.008]. Hemoglobin levels were also lower in the $\mathrm{AF}$ group [12.7 (2.2) vs. 13.7 (1.9) gr/dL, $\mathrm{p}=0.004]$. A greater number of patients in the AF group were classified as Killip class 3 and 4 at admission (3.9\% vs. $2.9 \%, \mathrm{p}=0.001)$. Severe mitral regurgitation was more common in AF patients $(11.7 \%$ vs. $1.7 \%, \mathrm{p}<0.001)$. Although the STEMI rate was $46.0 \%$ for the entire study population, only $3.0 \%$ of the total population, none of which were in the AF group, received thrombolytic treatment as a reperfusion therapy before diagnostic coronary angiography. Oral $\mathrm{P} 2 \mathrm{Y}_{12}$ inhibitor loading before the index emergency admission rate was higher in AF patients (96.0\% vs. 91.8\%, $\mathrm{p}=0.027)$. Clopidogrel was the most commonly used $\mathrm{P}_{2} \mathrm{Y}_{12}$ inhibitor in the study population $(49.2 \%)$. On the other hand, the loading rate of oral $\mathrm{P}_{2} \mathrm{Y}_{12}$ inhibitors other than clopidogrel was $31.3 \%$ in the $\mathrm{AF}$ and $47.6 \%$ in the non-AF group $(\mathrm{p}=0.004)$. Preexisting oral anticoagulant use was also higher in $\mathrm{AF}$ patients $(19.6 \%$ vs. $1.0 \%, \mathrm{p}<0.001)$ (Table 2$)$.

Coronary angiography results:

The majority of patients were treated via primary percutaneous intervention (1280 patients, $78.7 \%$ of the overall study population). Coronary artery bypass grafting was the preferred method for revascularization for $10.5 \%$ of cases and medical management was preferred for $10.2 \%$. The selected treatment options were similar between the AF and non-AF patients (Table 3). The percentages of patients diagnosed with MINOCA were also similar between gropus $(7.8 \%$ vs. $6.6 \%, \mathrm{p}=0.743)$.

\section{Discussion}

In the current study investigating the prevalence and demographic and clinical characteristics of $\mathrm{AF}$ in patients with de novo myocardial infarction, we found a lower prevalence rate of $3.1 \%$, in contrast to previous studies in the literature. ${ }^{1,2}$ The exclusion of patients with a previous history of revascularization and diagnosis of stable coronary artery disease (SCAD) as well as the relatively younger age of the enrolled population is thought to play a main role in the lower than expected prevalence of AF in our study. SCAD and AF share common risk factors and patients with a long history of SCAD and its risk factors also show increased risk of incident AF. ${ }^{5}$ In addition, coronary revascularization procedures, particularly CABG, have been found to create a predisposition to incident AF ${ }^{6}$ For this reason, the authors are of the opinion that the exclusion of patients with SCAD and revascularization history were the main factors behind the low percentage of AF patients in the study population. Furthermore, a positive correlation between age and AF has been confirmed in many registries. ${ }^{7}$ The relatively young mean age (approximately 60 years old) of patients enrolled in our study may also have resulted in the lower prevalence of AF in our study population. Theoretically, patients without previous diagnosis of SCAD and/or without history of revascularization may be younger and have fewer AF risk factors. Although ethnicity may play a role for differences in the prevalence of AF, no evidence has been reported showing different rates of AF between Turkey and the rest of the world. ${ }^{8}$ However, acute coronary syndrome registries conducted in Turkey report younger average ages in patients admitted with acute MI than in European countries and the United States. ${ }^{9,10}$ We believe that the younger age pattern may have an effect on lower AF prevalence in our study than that of other global registries.

Our study also found that non-ST-elevation myocardial infarction (NSTEMI) presentation was higher in the $\mathrm{AF}$ population than in patients without $\mathrm{AF}$, with approximately $70 \%$ of $\mathrm{AF}$ patients presenting with 
NSTEMI. Age is a common risk factor of both AF and NSTEMI. The current literature shows a positive correlation between increasing age and the rate of NSTEMI in the MI population and a higher prevalence of AF in patients with non-ST segment elevation acute coronary syndrome (NSTE-ACS). ${ }^{11}$ On the other hand, AF with fast ventricular response can precipitate Type $2 \mathrm{MI}$ that commonly presents with ST segment changes rather than elevation on admission ECG. ${ }^{1}$ However, while this study did not find any differences in the prevalence of MINOCA between the AF and non-AF groups, possible type II error cannot be ignored.

Advanced age, female gender, lower LV ejection fraction (LVEF), higher Killip class, obesity, presence of multiple comorbidities, and hemodynamic instability are associated with $\mathrm{AF} .{ }^{2}$ Similar to previous studies, patients in the AF group were older than in the non-AF group and the proportion of women to men in the AF group was higher than that in the non-AF group. Previous registries revealed that with advanced age, gender difference in MI patients lessens and the female/male ratio increases. ${ }^{12}$ AF patients were an average of twelve years older than non-AF patients in our study, which may explain the gender difference between the AF and non-AF groups. Additionally, smoking is more common in men than in women in the Turkish population. ${ }^{13}$ The authors believe that the observed difference in smoking between the groups may be due to the female dominance in the AF group. Hypertension is an essential predictor of AF development ${ }^{14}$ and higher hypertension prevalence in the $\mathrm{AF}$ group can play a role in the development of $\mathrm{AF}$ in advanced age ( $64.7 \%$ vs. $48.5 \%, p=0.023)$. In addition, we should pay attention to type I statistical error in evaluations of differences of gender, smoking, and prevalence of hypertension in this context. Lower LVEF in the AF group (45.0\% [30.0-50.0] vs. 50.0\% [35.0-55.0], $\mathrm{p}=0.008)$ can be explained by the higher burden of comorbidities such as advanced age and hypertension in AF patients. Loss of atrial kick and possible tachycardiomyopathy are other factors that may result in lower EF and have been found to be responsible for worse hemodynamic status and increased mortality. ${ }^{15}$ Accordingly, the ratio of Killip class 3 and 4 patients at admission was higher in the AF group in our study.

An interesting finding in our study was the relatively low percentage of patients who were initially treated by the thrombolytic therapy. ST-elevation myocardial infarction (STEMI) patients made up nearly half of the study population (46.0\%) but only $3.0 \%$ of all patients received thrombolytic treatment before coronary angiography. Even in the AF group, while $31.3 \%$ of patients presented with STEMI, none received thrombolytic therapy. It is possible that the lower preference for thrombolytic therapy as an initial reperfusion choice may be related to the well-organized ambulance system and sufficient number of invasive cardiology centers which are capable of performing $7 / 24$ primary PCI around the country.

Oral $\mathrm{P}_{2} \mathrm{Y}_{12}$ inhibitor loading was common in both $\mathrm{AF}$ and non-AF patients. Although the AF group was found to have a higher oral $\mathrm{P}_{2} \mathrm{Y}_{12}$ inhibitor loading rate $(96.0 \%$ vs. $91.8 \%, \mathrm{p}=0.027)$ than the non-AF group, this can be an incidental finding and accepted as an example of type I statistical error. The study also found the use of oral $\mathrm{P}_{2} \mathrm{Y}_{12}$ inhibitors other than clopidogrel to be relatively common in the AF group, with approximately one third of patients in the AF group loaded with an oral $\mathrm{P} 2 \mathrm{Y}_{12}$ inhibitor other than clopidogrel. We believe that the relatively common use of more potent $\mathrm{P}^{2} \mathrm{Y}_{12}$ inhibitors in AF population was due to the lower percentage of AF patients who had been already taken OAC therapy. It can be argued that with common $\mathrm{OAC}$ usage, potent $\mathrm{P}^{2} \mathrm{Y}_{12}$ inhibitor loading ratios would be lower.

Ischemic stroke is a devastating complication of $\mathrm{AF}$ and $\mathrm{MI}$ patients with $\mathrm{AF}$ carry a significant risk of stroke. Previously, it has been reported that dual/triple therapy by adding an OAC decreases the risk of stroke. ${ }^{16}$ Despite this stroke risk, most AF patients admitted with MI were discharged without any OAC therapy. Many trials have focused on finding the sweet spot between stroke, stent thrombosis, and major bleeding risk and compared triple and dual therapy strategies with different duration and different regimens. Almost all of reported greater safety with dual therapy with OAC plus clopidogrel than triple therapy. ${ }^{17}$ Current guidelines advise personalized management of these patients according to bleeding and thrombosis risk. ${ }^{18,19}$ It is also important to emphasize the low percentage of oral anticoagulant usage in patients with AF before emergency admission.

Underdiagnosed/overlooked AF, particularly in asymptomatic patients, undertreatment, and compliance issues with OAC therapy may be the main reasons for the lower percentage of OAC use in the study 
population. These results show the importance of successful and timely diagnosis of AF, patient education on OAC therapy, and transparent performance metrics and guideline adaptation for health providers.

In conclusion, the study reports a lower percentage of AF prevalence in the de novo MI population than previous studies of enrolled unselected MI and ACS cohorts. It also demonstrated the underuse of OAC in $\mathrm{AF}$ patients, emphasizing the vital role of opportunistic diagnostic strategies, patient education, and implementations for guideline adaptation.

Study limitations: The study had several limitations discussed below. Regarding the cross-sectional design of the study, it was not possible to obtain short and long-term prognostic metrics of the patient population. Due to the relatively small sample size of the AF population, possible type II statistical errors may have affected the comparative data. Some demographic and clinical characteristics of the study population may be specific to the Turkish population and thus not reflect a global perspective. MI was defined in line with the Third Universal Definition of Myocardial Infarction. However, the current Fourth Universal Definition of Myocardial Infarction was published after the starting date of study and protocol revisions were not made due to possible risks of harmonization between pre- and post-revision data. AF diagnosis was based on a12 lead ECG performed at admission, meaning that incident AF could not be captured and differentiated from existing chronic patterns. For this reason, the organizing committee required the completion of a form from the investigators in case with suspicion of incident AF. Similarly, we did not receive any data about the patterns of AF (ie first diagnosed, paroxysmal, persistent, etc). A higher rate of first diagnosed AF patterns may explain the dramatic percentages of OAC use in the study population.

Author contributions: Concept: A.C, U.O.T.; Design: U.O.T., A.C.; Supervision: U.O.T., M.Z., O.E.; Materials: A.C., C.E., G.A., Y.D., O.A.O., S.K., Y.C., I.U., M.B.T., R.D., V.P., H.O., M.O., K.T., D.K., N.C., M.G., S.I., F.Y.C., H.A., A.A., M.Z., O.E., U.O.T.; Data: A.C., S.K.; Analysis: A.C., S.K., U.O.T.; Literature search: A.C., U.O.T; Writing: A.C., U.O.T; Critical revision: U.O.T., M.Z., O.E.

Funding: This work was supported by the Cardiovascular Academy Society of Turkey [grant number: KVAK 2018/01].

Acknowledgements: The study was presented at European Society of Cardiology Congress, Paris 2019.

Conflict of interest: none declared.

\section{References}

1. Kalarus Z, Svendsen JH, Capodanno D, et al. Cardiac arrhythmias in the emergency settings of acute coronary syndrome and revascularization: an European Heart Rhytm Association (EHRA) consensus document, endorsed by the European Association of Percutaneous Cardiovascular Interventions (EAPCI), and European Acute Cardiovascular Care Association (ACCA). Europace. 2019;21:1603-1604.

2. Ibanez B, James S, Agewall S, et al. 2017 ESC Guidelines for the management of acute myocardial infarction in patients presenting with ST-segment elevation: The task Force for the management of acute myocardial infarction in patients presenting with ST-segment elevation of the European Society of Cardiology (ESC). Eur Heart J . 2018;39:119-177.

3. Kober L, Swedberg K, McMurray JJ, et al. Previously known and newly diagnosed atrial fibrillation: a major risk indicator after a myocardial infarction complicated by heart failure or left ventricular dysfunction. Eur J Heart Fail. 2006;8:591-598.

4. Türk UÖ, Zoghi M, Alioğlu E, Ergene O. Rationale and design of the myocardial infarction with nonobstructive coronary arteries in Turkish population (MINOCA-TR) study. Turk Kardiyol Dern Ars . 2019;47:662-668.

5. Yasuda S, Kaikita K, Akao M, et al; AFIRE Investigators. Antithrombotic therapy for atrial fibrillation with stable coronary disease. N Engl J Med. 2019;381:1103-1113.

6. Banach M, Rysz J, Drozdz JA, et al. Risk factors of atrial fibrillation following coronary artery bypass grafting: A preliminary report. Circ J. 2006;70:438-441. 
7. Rathore SS, Berger AK, Weinfurt KP, et al. Acute myocardial infarction complicated by atrial fibrillation in the elderly: prevalence and outcomes. Circulation. 2000;101:969-974.

8. Yavuz B, Ata N, Oto E, et al. Demographics, treatment and outcomes of atrial fibrillation in a developing country: the population-based TuRkish Atrial Fibrillation (TRAF) cohort. Europace.2017;19:734740 .

9. Ertaş FS, Tokgözoğlu L; EPICOR Study Group. Pre- and in-hospital antithrombotic management patterns and in-hospital outcomes in patients with acute coronary syndrome: data from the Turkish arm of the EPICOR study. Anatol J Cardiol. 2016;16:900-915.

10. Awad HH, Zubaid M, Alsheikh-Ali AA, et al. Comparison of characteristics, management practices, and outcomes of patients between the global registry and the gulf registry of acute coronary events. Am J Cardiol. 2011;108:1252-1258.

11. Mohamed MO, Kirchhof P, Vidovich M, et al. Effect of concomitant atrial fibrillation on in-hospital outcomes of non-ST-elevation acute coronary syndrome-related hospitalizations in the United States. Am J Cardiol. 2019;124:465-475.

12. Mehta LS, Beckie TM, DeVon HA, et al. Acute myocardial infarction in women: a scientific statement from the American Heart Association. Circulation. 2016;133:916-947.

13. Kilic D, Ozturk S. Gender differences in cigarette consumption in Turkey: evidence from the Global Adult Tobacco Survey. Health Policy. 2014;114:207-214.

14. Kallistratos MS, Poulimenos LE, Manolis AJ. Atrial fibrillation and arterial hypertension. Pharmacol Res. 2018;128:322-326.

15. Namana V, Gupta SS, Sabharwal N, Hollander G. Clinical significance of atrial kick. QJM. 2018;111:569-570.

16. Saito Y, Kobayashi Y. Triple therapy: A review of antithrombotic treatment for patients with atrial fibrillation undergoing percutaneous coronary intervention. J Cardiol. 2019;73:1-6.

17. Lopes RD, Hong H, Harskamp RE, et al. Optimal antithrombotic regimens for patients with atrial fibrillation undergoing percutaneous coronary intervention: an updated network meta-analysis. JAMA Cardiol.2020;5:1-8.

18. January CT, Wann LS, Calkins H, et al. 2019 AHA/ACC/HRS focused update of the 2014 AHA/ACC/HRS guideline for the management of patients with atrial fibrillation: A report of the American College of Cardiology/American Heart Association task force on clinical practice guidelines and the Heart Rhythm Society in collaboration with the Society of Thoracic Surgeons. Circulation. 2019;140:e125-e151.

19. Steffel J, Verhamme P, Potpara TS, et al; ESC Scientific Document Group. The 2018 European Heart Rhythm Association practical guide on the use of non-vitamin K antagonist oral anticoagulants in patients with atrial fibrillation. Eur Heart J. 2018;39:1330-1393.

Table-1: Demographic and clinical characteristics of study population at admission.

\begin{tabular}{|c|c|c|c|}
\hline & AF patients $(n=51)$ & $\begin{array}{l}\text { non-AF patients } \\
(\mathrm{n}=1575)\end{array}$ & $\mathrm{p}$ value \\
\hline $\begin{array}{l}\text { Mean age (years), } \\
\text { mean (SD) }\end{array}$ & $73.4(9.4)$ & $61.0(12.4)$ & $<0.001$ \\
\hline $\begin{array}{l}\text { Female gender, } \mathbf{n} \\
(\%)\end{array}$ & $22(43.1)$ & $453(28.7)$ & 0.027 \\
\hline $\begin{array}{l}\text { BMI }\left(\mathrm{kg} / \mathrm{m}^{2}\right), \text { mean } \\
(\mathrm{SD})\end{array}$ & $26.9(4.1)$ & $27.6(4.4)$ & 0.522 \\
\hline Smoking, n (\%) & $20(39.2)$ & $922(58.6)$ & 0.006 \\
\hline $\begin{array}{l}\text { Diabetes mellitus, } \mathbf{n} \\
(\%)\end{array}$ & $20(39.2)$ & $460(30.3)$ & 0.174 \\
\hline Hypertension, n (\%) & $33(64.7)$ & $744(48.5)$ & 0.023 \\
\hline $\begin{array}{l}\text { Hyperlipidemia, } \mathbf{n} \\
(\%)\end{array}$ & $15(32.6)$ & $482(35.9)$ & 0.640 \\
\hline
\end{tabular}




\begin{tabular}{|c|c|c|c|}
\hline & AF patients $(n=51)$ & $\begin{array}{l}\text { non-AF patients } \\
(\mathrm{n}=1575)\end{array}$ & $p$ value \\
\hline $\begin{array}{l}\text { Systolic blood } \\
\text { pressure (mmHg), } \\
\text { mean (SD) }\end{array}$ & $130.9(27.3)$ & $128.9(23.3)$ & 0.872 \\
\hline $\begin{array}{l}\text { Diastolic blood } \\
\text { pressure }(\mathrm{mmHg}), \\
\text { mean (SD) }\end{array}$ & $78.2(16.2)$ & $77.3(13.9)$ & 0.761 \\
\hline $\begin{array}{l}\text { Admission KILLIP } \\
\text { class ([?]3), n (\%) }\end{array}$ & $2(3.9)$ & $47(2.9)$ & 0.001 \\
\hline $\begin{array}{l}\text { LV ejection fraction } \\
(\%), \text { median } \\
\left(25^{\text {th }}-75^{\text {th }} \text { percentile }\right)\end{array}$ & $45.0(30.0-50.0)$ & $50.0(35.0-55.0)$ & 0.008 \\
\hline $\begin{array}{l}\text { Presence of severe } \\
\left([?] 3^{\text {rd }} \text { degree }\right) \mathrm{MR}, \\
\mathbf{n}(\%)\end{array}$ & $6(11.7)$ & $27(1.7)$ & $<0.001$ \\
\hline $\begin{array}{l}\text { Blood glucose } \\
(\mathrm{mg} / \mathrm{dL}), \text { median } \\
\left(25^{\text {th }}-75^{\text {th }} \text { percentile }\right)\end{array}$ & $140.0(85.0-192.0)$ & $123.0(81.0-176.0)$ & 0.086 \\
\hline $\begin{array}{l}\text { Creatinine }(\mathrm{mg} / \mathrm{dL}) \\
\text { median }\left(25^{\text {th }}-75^{\text {th }}\right. \\
\text { percentile })\end{array}$ & $0.90(0.71-1.50)$ & $0.85(0.67-1.31)$ & 0.203 \\
\hline $\begin{array}{l}\text { Hemoglobin } \\
\text { (gr/dL), mean (SD) }\end{array}$ & $12.7(2.2)$ & $13.6(1.9)$ & 0.004 \\
\hline $\begin{array}{l}\text { hs-Troponin }(\mathrm{ng} / \mathrm{mL}) \text {, } \\
\text { median }\left(25^{\text {th }}-75^{\text {th }}\right. \\
\text { percentile })\end{array}$ & $15.2(1.4-97.1)$ & $15.3(0.9-428.0)$ & 0.689 \\
\hline $\begin{array}{l}\text { NSTEMI } \\
\text { presentation, } \mathbf{n}(\%)\end{array}$ & $35(68.6)$ & $839(53.2)$ & 0.023 \\
\hline
\end{tabular}

Abbreviations: BMI, body mass index; LV, left ventricule; MR, mitral regurgitation

Table-2: Percentages of oral $\mathrm{P}_{2} \mathrm{Y}_{12}$ loading at admission and preexisting use of oral anticoagulant

\begin{tabular}{llll}
\hline & AF patients $(\mathbf{n = 5 1 )}$ & $\begin{array}{l}\text { non-AF patients } \\
(\mathbf{n = 1 5 7 5 )}\end{array}$ & p value \\
\hline $\begin{array}{l}\text { P2Y }{ }_{12} \text { inhibitors } \\
\text { loading, }(\%)\end{array}$ & $49(96.0 \%)$ & $1446(91.8 \%)$ & 0.027 \\
$\begin{array}{l}\text { Preexisting oral } \\
\text { anticoagulant use, } \mathrm{n}\end{array}$ & $10(19.6 \%)$ & $16(1.0 \%)$ & $<0.001$ \\
$(\%)$ & & & \\
\hline
\end{tabular}

Table-3: Preferred treatment strategies for coronary artery disease $(\mathrm{p}=0.884)$

\begin{tabular}{lll}
\hline & AF patients $(\mathbf{n}=\mathbf{5 1})$ & non-AF patients $(\mathbf{n}=\mathbf{1 5 7 5})$ \\
\hline Percutaneous coronary intervention (PCI), n (\%) & $38(74.5 \%)$ & $1244(79.0 \%)$ \\
Coronary artery bypass grafting (CABG), n (\%) & $5(9.8 \%)$ & $166(10.6 \%)$ \\
Medical management, n (\%) & $8(15.7 \%)$ & $158(10.0 \%)$
\end{tabular}


AF patients $(n=51) \quad$ non-AF patients $(n=1575)$

Other options, n (\%)

$0(0.0 \%)$

$7(0.4 \%)$

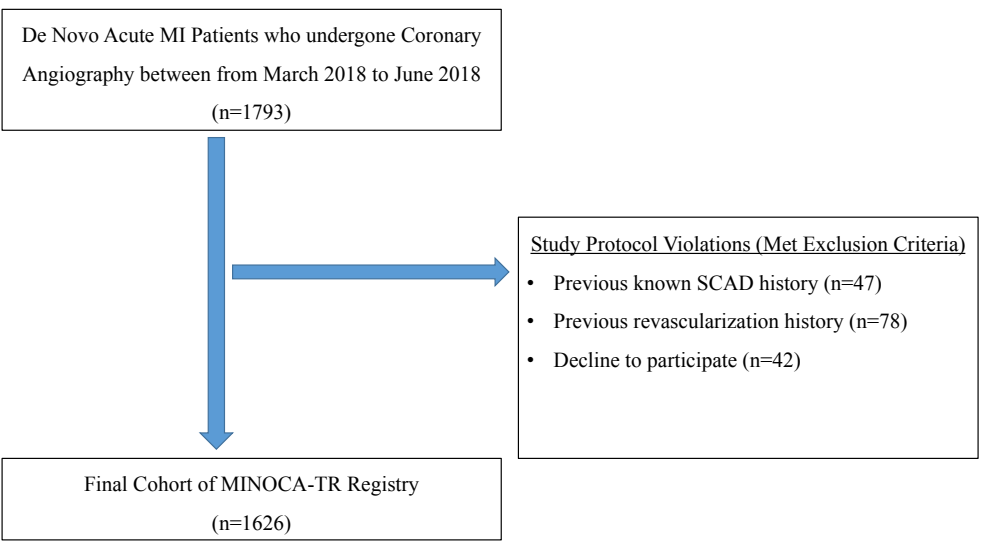

Figure-1: Derivation of the study cohort. MI, myocardial infarction; SCAD, stable coronary artery disease. 